\title{
Parameter dependence of the topology change and the scaling properties of the topology conserving gauge action
}

\author{
Hidenori Fukaya, Tetsuya Onogi* \\ Yukawa Institute for Theoretical Physics, Kyoto University, Kyoto 606-8502, Japan.

\section{Shoji Hashimoto} \\ High Energy Accelerator Research Organization (KEK), Tsukuba 305-0801, Japan. \\ School of High Energy Accelerator Science, The Graduate University for Advanced Studies \\ (Sokendai), Tsukuba 305-0801, Japan.
}

\section{Takuya Hirohashi}

Department of Physics, Kyoto University, Kyoto 606-8502, Japan.

\section{Kenji Ogawa}

School of High Energy Accelerator Science, The Graduate University for Advanced Studies (Sokendai), Tsukuba 305-0801, Japan.

\begin{abstract}
The topology conserving gauge action proposed by Lüscher is expected to reduce the number of non-smooth gauge configurations as well as the topology change compared to the conventional actions for the same lattice spacings. We report our quenched QCD study of the topological stability and the scaling violation of the static quark potential. We find that the the topology change is indeed suppressed when the parameter $\varepsilon$ is of order one. We also find that the scaling violation in the static quark potential remain reasonably small in the parameter range of our study. Our study is done at the inverse lattice spacing $a^{-1}=1.4-2.5 \mathrm{GeV}$ with the lattice size $L=1.0$ $1.6 \mathrm{fm}$.
\end{abstract}

XXIIIrd International Symposium on Lattice Field Theory

25-30 July 2005

Trinity College, Dublin, Ireland

\footnotetext{
*Speaker.
} 


\section{Introduction}

The admissibility condition of the gauge fields on the lattice $[1,2]$

$$
\left\|1-P_{\mu v}(x)\right\|<\varepsilon \text { for all } x, \mu, v,
$$

guarantees the locality and the uniqueness of the index of the overlap-Dirac operator. [3, 4]. As an example, the gauge action which restricts the gauge field to satisfy the bound (1.1)

$$
S_{G}=\beta \sum_{P} \frac{1-\operatorname{Re} \operatorname{Tr} P_{\mu v}(x) / 3}{1-\left(1-\operatorname{Re} \operatorname{Tr} P_{\mu v}(x) / 3\right) / \varepsilon},
$$

was proposed by Lüescher [1]. This action may be useful for QCD simulations in two reasons: (1) it may serve to efficiently collect gauge configurations with fixed topology $Q \neq 0$ in the $\varepsilon$-regime, (2) it may give a possibility to reduce the numerical cost of dynamical overlap fermions with fewer low-lying modes of $H_{W}$ and less frequent topology change. In fact, this action has been proven to be useful for the massive Schwinger model for stabilizing the topological charge and for improving the chiral behavior of the domain wall fermions [5,6]. Also in the four-dimensional quenched QCD, the good stability of the topological charge has been observed with some reasonable choices of parameters [7, 8, 9]. See also [10].

In this report we present our quenched study of the topology conserving gauge action (1.2) on the stability of the topology and the scaling. Studies on the low-mode distribution in quenched and dynamical QCD are reported by Matsufuru [11].

\section{Lattice setup}

In our study, we take three values of $1 / \varepsilon(=0,2 / 3$, and 1$)$ with three lattice sizes $12^{4}, 16^{4}$, and $20^{4}$ and four lattice spacings in the range $a^{-1}=1.4-2.5 \mathrm{GeV}$. Here, $1 / \varepsilon=0$ corresponds to the conventional plaquette action and $1 / \varepsilon=2 / 3$ is the boundary, below which, all gauge configurations are allowed when the gauge group is $S U(3)$. Link variables are generated by the standard hybrid Monte Carlo algorithm with $\Delta \tau=0.01-0.02$ and $N_{m d s}=20-40$, where $N_{m d s}$ is the number of molecular dynamics steps and $\Delta \tau$ denotes its step-size. We accumulated at least 2,000 trajectories for thermalization before measuring observables. We monitored the plaquette values of the gauge fields, but we did observe any case where the admissibility condition is violated through the hybrid Monte Carlo updates.

\section{Stability of the topological charge}

In order to measure the topological charge, we developed a new cooling method, in which we carry out the hybrid Monte Carlo simulation using the topology conserving action with an exponentially increasing coupling $\beta=\beta_{\text {cool }}$ and an exponentially decreasing step size $\Delta \tau$ as functions of trajectory $n_{t}$. After 50-200 steps, the gauge fields are expected to be cooled down close to the classical background in each topological sector. In fact, the geometrical definition of the topological charge [12]

$$
Q_{g e o} \equiv \frac{1}{32 \pi^{2}} \sum_{x} \varepsilon^{\mu v \rho \sigma} \operatorname{Re} \operatorname{Tr}\left(P_{\mu v}(x) P_{\rho \sigma}(x)\right)
$$




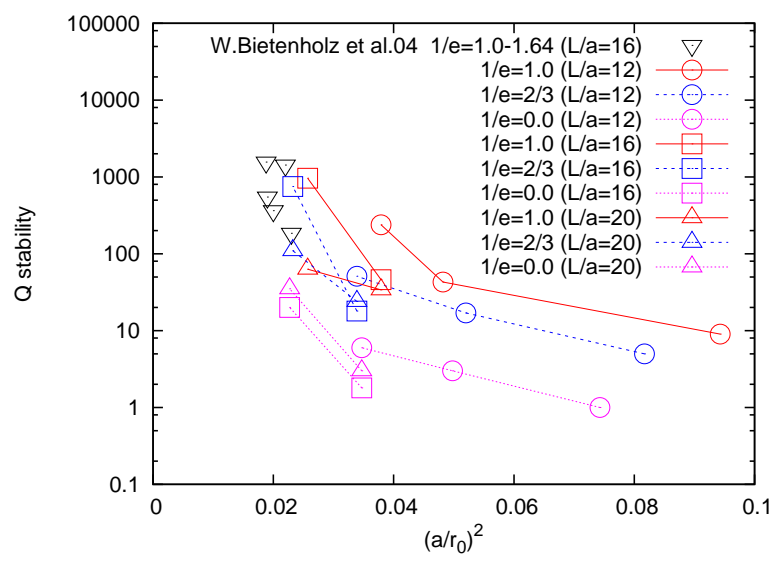

Figure 1: Stability of the topological charge (3.1) for each parameter set. Results are plotted as a function of lattice spacing squared. Three values of $1 / \varepsilon$ are distinguished by lines: 0 (dotted), 2/3 (dashed), 1 (solid). Circles, squares, and up-triangles correspond to lattice sizes 12, 16 and 20, respectively. The down-triangles are from [8].

of these "cooled" configurations gives numbers close to an integer.

Figure 1 shows the stability of the topological charge defined as

$$
\operatorname{Stab}_{Q} \equiv \frac{N_{\text {trj }}}{\tau_{\text {plaq }} \times \# Q}
$$

where $\tau_{\text {plaq }}$ is the autocorrelation time of the plaquette, measured by the method in Appendix $\mathrm{E}$ of [13], $N_{\text {trj }}$ denotes the number of trajectories and $\# Q$ is the number of topology changes. Since the topological charge is measured every $10-20$ trajectories, $\mathrm{Stab}_{Q}$ gives only an upper limit. Our results show that the topological charge becomes more stable for higher $1 / \varepsilon$, smaller $L$ and smaller a.

\section{Static quark potential}

We calculate the static quark potential $V(\vec{r})$ from the Wilson loops $W(\vec{r}, t)$ for every 20 trajectories. The spatial side $\vec{r}$ is taken to be in 6 different directions of the 3-dimensional unit vectors: $\vec{u}$ $=(1,0,0),(1,1,0),(2,1,0),(1,1,1),(2,1,1),(2,2,1)$. We calculate the Sommer scales $r_{0}$ and $r_{c}$ defined as $r_{0}^{2} F\left(r_{0}\right)=1.65$ and $r_{c}^{2} F\left(r_{c}\right)=0.65$, respectively $[14,15] . F$ is the force obtained from the static quark potential. We choose a $Q=0$ configuration for the initial condition of HMC steps and do not take care of the topological charge hysteresis assuming that the topology of the gauge fields would not affect the Wilson loops if they are small enough.

Figure 2 shows the scaling of $r_{c} / r_{0}$. One can see that they agree well with the results with the plaquette action and its continuum limit [15] in the region $\left(a / r_{0}\right)^{2} \lesssim 0.08$. Also, as seen in Figure 3 for long distances, the quark potential itself $\hat{V}(\vec{r})=r_{0}\left(V(\vec{r})-V\left(r_{c}\right)\right)$ does show a good agreement with that in the continuum limit obtained with the plaquette action [15]. Here, $V\left(r_{c}\right)$ is measured using an interpolation polynomial of order 5. For short distances, they show 10-20\% deviations due to the violation of the rotational symmetry but the discrepancies are comparable to those with the plaquette action. 


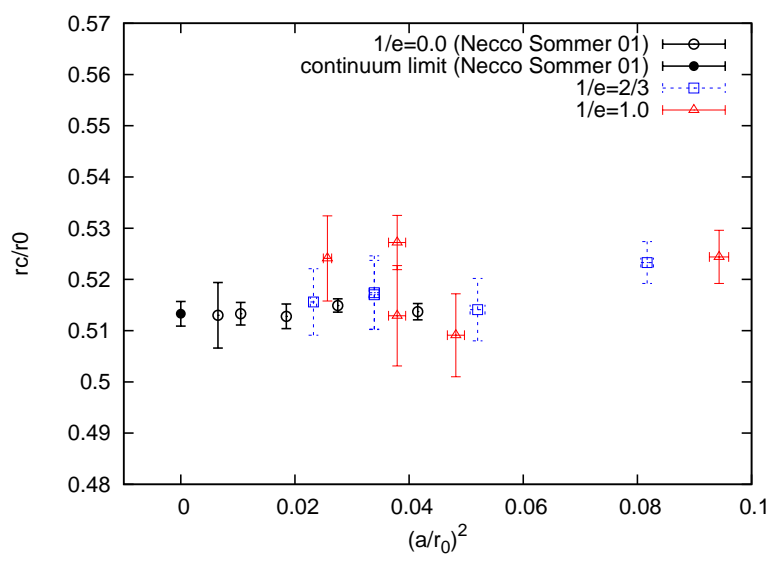

Figure 2: Scaling of $r_{c} / r_{0}$. Squares and triangles are data for $1 / \varepsilon=2 / 3$ and for 1 , respectively. Open circles are the result with the plaquette action in [15], while the filled circle denotes their continuum limit.
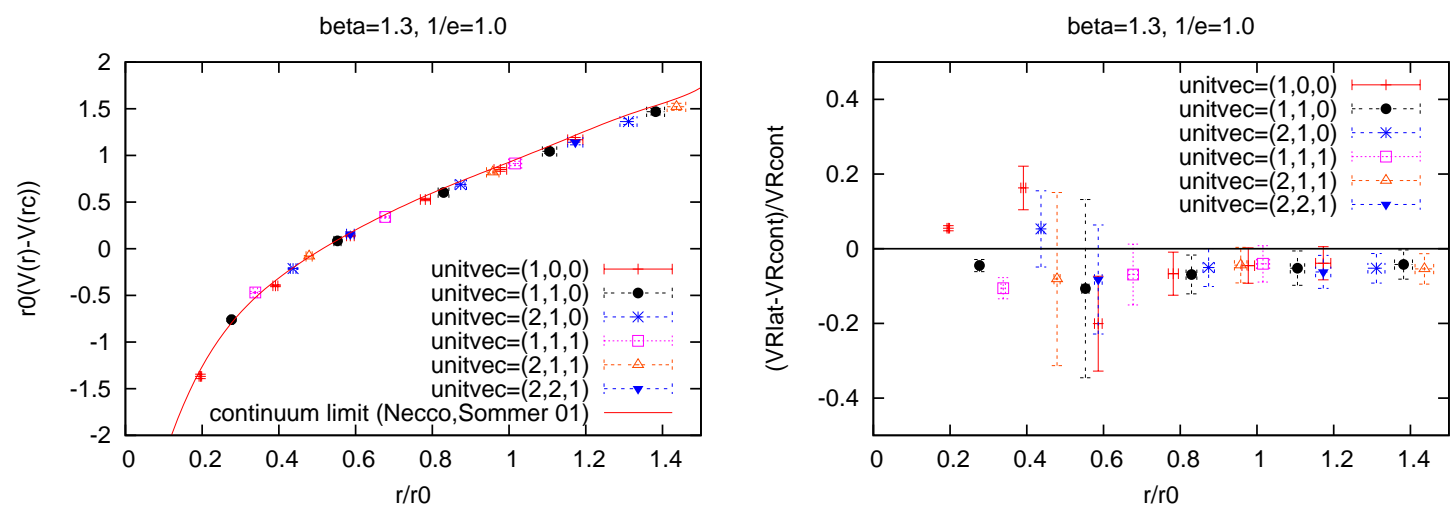

Figure 3: Left: heavy quark potential at $\beta=1.3,1 / \varepsilon=1.0$ on a $12^{4}$ lattice. Dashed line shows the continuum limit obtained by an interpolation from the result of [15]. Different symbols show the $V(\vec{r})$ 's with different orientations parallel to $\vec{u}$ 's. Right: $\left(\hat{V}(\vec{r})-\hat{V}_{\text {cont }}(\vec{r})\right) / \hat{V}_{\text {cont }}(\vec{r})$, where $\hat{V}_{\text {cont }}(\vec{r})$ represents the continuum limit. The error of $\hat{V}_{\text {cont }}(\vec{r})$ is ignored $(\lesssim 1 \%)$.

We conclude that the heavy quark potential for the admissible gauge fields is quite similar with that of the plaquette action and no serious inconsistency can be found.

\section{Perturbative renormalization of the coupling}

Ellis and Martinelli computed the two-loop corrections to the gauge coupling for general gauge actions which can be written by the plaquette [16]. Using their formula, the renormalized gauge couping $g_{M}$ in the so-called Manton scheme is expressed by the bare coupling $g_{0}$ as

$$
\frac{1}{g_{M}^{2}(1 / a)}=\frac{1}{g_{0}^{2}}+A_{1}+A_{2} g_{0}^{2}
$$

where $\left(A_{1}, A_{2}\right)=(-0.2083,-0.03056),(0.34722,-0.04783)$, and $(0.625,-0.10276)$ for $1 / \varepsilon=0$, $2 / 3$, and 1, respectively. In Figure 4 we plot the inverse squared coupling in the Manton scheme at a reference scale $\mu \equiv 5 / r_{0}$ as a function of the lattice spacing. Here, we use the one-loop formula 


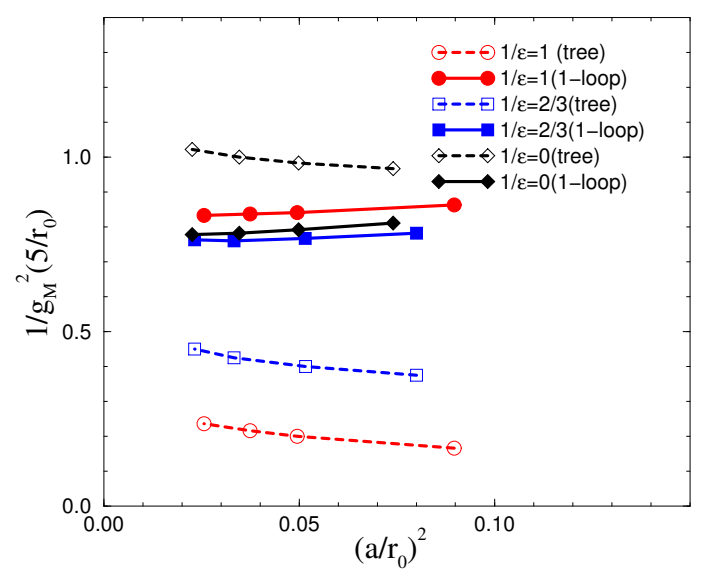

Figure 4: $1 / g_{M}^{2}\left(5 / r_{0}\right)$ in the Manton scheme as a function of lattice spacing squared. Results for various $1 / \varepsilon$ and $r_{0}$ are plotted. The bare coupling $1 / g_{0}^{2}$ is also shown by open symbols for comparison.

in (5.1) for the change of scheme and two-loop renormalization group equation for the evolution to the reference scale. We find that the renormalized coupling for various values of $1 / \varepsilon$ shows a good agreement, which means that the change of $1 / \varepsilon$ is well described by the perturbation theory. One can also see that the scaling violation is small for the renormalized coupling. However, we note that the two-loop result gives larger corrections for larger $1 / \varepsilon$ so that the convergence of the perturbative series are poor for $1 / \varepsilon=1$ and marginal for $1 / \varepsilon=2 / 3$.

\section{Summary}

We studied the topology conserving gauge action in the quenched approximation for various values of $\beta, 1 / \varepsilon$ and the lattice size. By measuring the topological charge with a new cooling method, we find that the stability of the topological charge is improved for larger $1 / \varepsilon$ when compared at a same lattice spacing and lattice size. Measuring also the static quark potential and the Sommer scales $r_{0}, r_{c}$, the scaling violation are found to be small. Our study shows that the topology conserving gauge action is feasible for QCD simulations. Practical applications of this action such as QCD in $\varepsilon$-regime and simulations with dynamical overlap fermions are underway.

\section{ACKNOWLEDGMENTS}

We would like to thank W. Bietenholz, L. Del Debbio, L. Giusti, M. Hamanaka, K. Jansen, H. Kajiura, Y. Kikukawa, M. Lüscher, H. Matsufuru, S. Shcheredin, H. Suzuki and T. Umeda for discussions. The numerical simulation was mainly carried out on the NEC SX-5 at Research Center for Nuclear Physics. We also acknowledge the Yukawa Institute for Theoretical Physics at Kyoto University, where this work was benefited from the discussions during the YITP-W-04-02 workshop on "LFT mini workshop". T. O. is supported by the Grant-in-Aid for Scientific research from the Ministry of Education, Culture, Sports, Science and Technology of Japan (Nos. 13135213, 16028210, 16540243). 


\section{References}

[1] M. Luscher, Abelian chiral gauge theories on the lattice with exact gauge invariance, Nucl. Phys. B 549, 295 (1999) [arXiv:hep-lat/9811032].

[2] P. Hernandez, K. Jansen and M. Luscher, Locality properties of Neuberger's lattice Dirac operator, Nucl. Phys. B 552, 363 (1999) [arXiv:hep-lat/9808010].

[3] H. Neuberger, Exactly massless quarks on the lattice, Phys. Lett. B 417, 141 (1998) [arXiv:hep-lat/9707022].

[4] H. Neuberger, More about exactly massless quarks on the lattice, Phys. Lett. B 427, 353 (1998) [arXiv:hep-lat/9801031].

[5] H. Fukaya and T. Onogi, Lattice study of the massive Schwinger model with $\theta$ term under Lüescher's 'admissibility' condition, Phys. Rev. D 68, 074503 (2003) [arXiv:hep-lat/0305004].

[6] H. Fukaya and T. Onogi, $\theta$ vacuum effects on the chiral condensation and the eta' meson correlators in the two-flavor massive $Q E D_{2}$ on the lattice, Phys. Rev. D 70, 054508 (2004) [arXiv:hep-lat/0403024].

[7] S. Shcheredin, W. Bietenholz, K. Jansen, K. I. Nagai, S. Necco and L. Scorzato, Testing a topology conserving gauge action in QCD, arXiv:hep-lat/0409073.

[8] W. Bietenholz, K. Jansen, K. I. Nagai, S. Necco, L. Scorzato and S. Shcheredin [XLF Collaboration], Lattice gauge actions for fixed topology, AIP Conf. Proc. 756, 248 (2005) [arXiv:hep-lat/0412017].

[9] S. Shcheredin, Simulations of lattice fermions with chiral symmetry in quantum chromodynamics, arXiv:hep-lat/0502001.

[10] K. Nagai et al. in these proceedings.

[11] H. Matsufuru et al. in these proceedings.

[12] J. Hoek, M. Teper and J. Waterhouse, Topological Fluctuations And Susceptibility In SU(3) Lattice Gauge Theory, Nucl. Phys. B 288, 589 (1987).

[13] M. Luscher, Schwarz-preconditioned HMC algorithm for two-flavour lattice QCD, arXiv:hep-lat/0409106.

[14] M. Guagnelli, R. Sommer and H. Wittig [ALPHA collaboration], Precision computation of a low-energy reference scale in quenched lattice QCD, Nucl. Phys. B 535, 389 (1998) [arXiv:hep-lat/9806005].

[15] S. Necco and R. Sommer, The $N_{f}=0$ heavy quark potential from short to intermediate distances, Nucl. Phys. B 622, 328 (2002) [arXiv:hep-lat/0108008].

[16] R. K. Ellis and G. Martinelli, Two loop corrections to the $\Lambda$ parameters of one plaquette actions, Nucl. Phys. B 235, 93 (1984) [Erratum-ibid. B 249, 750 (1985)]. 